\title{
UPPER SEMICONTINUOUS PERTURBATIONS OF $m$-ACCRETIVE OPERATORS AND DIFFERENTIAL INCLUSIONS WITH DISSIPATIVE RIGHT-HAND SIDE
}

\author{
DIETER BOTHE \\ Fachbereich 17 der Universität Paderborn \\ D-33095 Paderborn, Germany \\ E-mail: dieterb@uni-paderborn.de
}

1. Introduction. Let $X$ be a real Banach space and $A: D(A) \subset X \rightarrow 2^{X} \backslash\{\emptyset\}$ be $m$-accretive. In applications one often has to deal with operators of the type $A+F$. Therefore it is of interest to have sufficient conditions guaranteeing that this sum is $m$-accretive again. This problem has attracted many people; see [1], [10], [13] and [14], the references given there and also [2], [4] and [12].

Of particular interest to us is Theorem 1 in [1], saying that $A+F$ is $m$-accretive if $F: X \rightarrow X$ is continuous and accretive. In the first part of this paper, we extend this and related results to the case of multivalued perturbations. More precisely, we prove that if $F: \overline{D(A)} \rightarrow 2^{X} \backslash\{\emptyset\}$ is upper semicontinuous with compact convex values such that $A+F$ is accretive, then $A+F$ is $m$-accretive. This result proves useful in the second part of this paper where we obtain existence of strong solutions of the initial value problem

$$
u^{\prime} \in F(t, u) \quad \text { on } J=[0, a], u(0)=x_{0},
$$

if, among other assumptions, the $F(t, \cdot)$ are usc with compact convex values and satisfy a condition of dissipative type.

2. Preliminaries. In the sequel, $X$ will always be a real Banach space with norm $|\cdot|$. Then $2^{X} \backslash \emptyset$ denotes the set of all nonempty subsets of $X, B_{r}(x)$ is the open ball in $X$ with center $x$ and radius $r, \bar{B}_{r}(x)$ denotes its closure and $\rho(x, B)$ is the distance from $x$ to the set $B \subset X$. Given $J=[0, a] \subset \mathbb{R}$, we let $C_{X}(J)$ be the Banach space

1991 Mathematics Subject Classification: Primary 47H06; Secondary 34A60.

The author gratefully acknowledges financial support by DAAD within the scope of the French-German project PROCOPE.

The paper is in final form and no version of it will be published elsewhere. 
of all continuous $u: J \rightarrow X$ and $L_{X}^{1}(J)$ the Banach space of all strongly measurable, Bochner-integrable $w: J \rightarrow X$, both equipped with the usual norms which we denote by $|\cdot|_{0}$, respectively $|\cdot|_{1}$. Given an operator $A: X \rightarrow 2^{X}$, we let $D(A)=\{x \in X \mid A x \neq \emptyset\}$, $R(A)=\bigcup_{x \in D(A)} A x$ and $\operatorname{gr}(A)=\{(x, y) \mid x \in D(A), y \in A x\}$ denote the domain, range and graph of $A$, respectively.

(i) Recall that $A: X \rightarrow 2^{X}$ is $m$-accretive if $R(A+\lambda I)=X$ for all $\lambda>0$ and $A$ is accretive, i.e.

$$
(u-v, x-y)_{+} \geq 0 \quad \text { for all } x, y \in D(A), u \in A x \text { and } v \in A y .
$$

Here $(\cdot, \cdot)_{+}$denotes one of the semi-inner products $(\cdot, \cdot)_{ \pm}$defined by

$$
(x, y)_{+}=|y| \lim _{t \rightarrow 0+} \frac{|y+t x|-|y|}{t} \quad \text { and } \quad(x, y)_{-}=|y| \lim _{t \rightarrow 0+} \frac{|y|-|y-t x|}{t} ;
$$

properties of $(\cdot, \cdot)_{ \pm}$can be found e.g. in $\S 4.4$ of [7]. If $A$ is $m$-accretive, the resolvents $J_{\lambda}:=(I+\lambda A)^{-1}: X \rightarrow D(A)$ and the Yosida-approximations $A_{\lambda}:=\lambda^{-1}\left(I-J_{\lambda}\right):$ $X \rightarrow X$ are well defined for all $\lambda>0$. In particular, $A_{\lambda} x \in A\left(J_{\lambda} x\right)$ on $X$, all $J_{\lambda}$ are nonexpansive mappings and $\lim _{\lambda \rightarrow 0+} J_{\lambda} x=x$ for every $x \in \overline{D(A)}$.

We shall use the following characterization of $m$-accretivity.

Lemma 1. Let $A$ be an accretive operator in $X$. Then $A$ is $m$-accretive if and only if $\operatorname{gr}(A)$ is closed and

$$
\underset{h \rightarrow 0+}{\lim _{h \rightarrow 0}} h^{-1} \rho(x+h z, R(I+h A))=0 \quad \text { for all } x \in \overline{D(A)} \text { and all } z \in X .
$$

This is Theorem 5.2 in [10]. More about $m$-accretive operators on Banach spaces can be found e.g. in [2] or [4]; in the latter reference one can also find Lemma 1 which is Theorem 16.2 there.

(ii) Let us also recall some facts about u.s.c. multivalued maps; for more details see [7]. A multivalued map $F: D \subset X \rightarrow 2^{X} \backslash \emptyset$ is called upper semicontinuous (u.s.c. for short), if $F^{-1}(B):=\{x \in D \mid F(x) \cap B \neq \emptyset\}$ is closed in $D$, for all closed $B \subset X$. If $F$ has compact values, u.s.c. is equivalent to: for every $\varepsilon>0$ and $x_{0} \in D$ there is $\delta=\delta\left(\varepsilon, x_{0}\right)>0$ such that $F(x) \subset F\left(x_{0}\right)+B_{\varepsilon}(0)$ on $B_{\delta}\left(x_{0}\right) \cap D$. A multivalued map is said to be continuous if it is continuous w.r. to the Hausdorff metric $d_{H}$ which is given by

for bounded $A, B \subset X$.

$$
d_{H}(A, B)=\max \left\{\sup _{x \in A} \rho(x, B), \sup _{x \in B} \rho(x, A)\right\}
$$

In case $D$ is compact and $F$ is u.s.c. with convex values, for every $\varepsilon>0$, there exists a continuous $f_{\varepsilon}: D \rightarrow X$ such that

$$
f_{\varepsilon}(x) \in F\left(B_{\varepsilon}(x) \cap D\right)+B_{\varepsilon}(0) \quad \text { on } D ;
$$

see Proposition 1.1 in [7]. Finally, the following fixed point theorem is a special case of Theorem 11.5 in [7]. 
Lemma 2. Let $X$ be a real Banach space, $\emptyset \neq D \subset X$ compact convex and $F: D \rightarrow$ $2^{D} \backslash \emptyset$ be u.s.c. with closed convex values. Then $F$ has a fixed point.

(iii) We also need the following criterion for weak relative compactness in $L_{X}^{1}(J)$.

Lemma 3. Let $X$ be a Banach space, $J=[0, a] \subset \mathbb{R}$ and $W \subset L_{X}^{1}(J)$ be uniformly integrable. Suppose that there exist weakly relatively compact sets $C(t) \subset X$ such that $w(t) \in C(t)$ a.e. on $J$, for all $w \in W$. Then $W$ is weakly relatively compact in $L_{X}^{1}(J)$.

This is Corollary 2.6 in [8] specialized to Lebesgue measure.

\section{Upper semicontinuous perturbations.}

Theorem 1. Let $X$ be a real Banach space, $A: D(A) \subset X \rightarrow 2^{X} \backslash \emptyset$ be m-accretive and $F: \overline{D(A)} \rightarrow 2^{X} \backslash \emptyset$ be u.s.c. with compact convex values such that $A+F$ is accretive. Then $A+F$ is $m$-accretive.

Pro of. Let $B=A+F$ with $D(B):=D(A)$. Then $B$ has closed graph, since $\left(x_{n}, y_{n}\right) \in$ $\operatorname{gr}(B)$ means $y_{n}=u_{n}+v_{n}$ with $u_{n} \in A x_{n}$ and $v_{n} \in F\left(x_{n}\right)$, hence $\left(x_{n}, y_{n}\right) \rightarrow(x, y)$ implies $v_{n} \in F(x)+B_{\varepsilon}(0)$ for all $n \geq n_{\varepsilon}$ and therefore $v_{n_{k}} \rightarrow v \in F(x)$ for some subsequence $\left(v_{n_{k}}\right)$ of $\left(v_{n}\right)$, hence also $u_{n_{k}} \rightarrow u:=y-v$ and $u \in A x$ by closedness of $\operatorname{gr}(A)$.

Next, notice that in order to get (2) we may assume $z=0$, since for any $z \in X$ the map $F_{z}$, defined by $F_{z}(x):=F(x)-\{z\}$ on $\overline{D(A)}$, has the same properties as $F$. So we are done by Lemma 1 , if

$$
\lim _{h \rightarrow 0+} h^{-1} \rho(x, R(I+h B))=0 \quad \text { on } \overline{D(B)} .
$$

Fix $x \in \overline{D(B)}$, let $h>0, C:=F(x)$ and $G(z):=F\left(J_{h}(x-h z)\right)$ for $z \in X$ where $J_{h}=(I+h A)^{-1}$. Evidently, $G$ is u.s.c. with compact convex values. Hence, given $\varepsilon>0$, the approximation result mentioned in 2. (ii) yields a continuous $g_{\varepsilon}: C \rightarrow X$ such that $g_{\varepsilon}(z) \in G\left(B_{\varepsilon}(z) \cap C\right)+B_{\varepsilon}(0)$ on $C$. Let $G_{\varepsilon}(z)=P_{C}\left(g_{\varepsilon}(z)\right)$ for $z \in C$, where $P_{C}(\cdot)$ is the metric projection onto $C$, i.e.

$$
P_{C}(x)=\{y \in C|| x-y \mid=\rho(x, C)\} \quad \text { on } X .
$$

Then $G_{\varepsilon}: C \rightarrow 2^{C} \backslash \emptyset$ is also u.s.c. with compact convex values, since $P_{C}$ has this properties. Therefore, $G_{\varepsilon}$ has a fixed point $z_{\varepsilon} \in C$ by Lemma 2. Given $h_{n} \searrow 0$ and $\varepsilon_{n} \searrow 0$ we repeat the previous arguments to obtain fixed points $z_{n}$ of the corresponding $G_{\varepsilon_{n}}$, i.e. we get a sequence $\left(z_{n}\right) \subset C$ such that

$$
z_{n} \in P_{C}\left(y_{n}\right) \quad \text { and } \quad y_{n} \in F\left(J_{h_{n}}\left(x-h_{n}\left(B_{\varepsilon_{n}}\left(z_{n}\right) \cap C\right)\right)\right)+B_{\varepsilon_{n}}(0) .
$$

In particular, there are $e_{n}, \widehat{e}_{n} \in B_{\varepsilon_{n}}(0)$ such that

$$
y_{n}-e_{n} \in F\left(J_{h_{n}}\left(x-h_{n} \widehat{z}_{n}\right)\right) \quad \text { with } \widehat{z}_{n}=z_{n}+\widehat{e}_{n} \in C .
$$

Now $x_{n}:=J_{h_{n}}\left(x-h_{n} \widehat{z}_{n}\right)$ satisfies $\left|x_{n}-x\right| \leq h_{n}\left|\widehat{z}_{n}\right|+\left|J_{h_{n}}(x)-x\right|$, i.e. $x_{n} \rightarrow x$ as $n \rightarrow \infty$. We may therefore assume $y_{n} \rightarrow y$ for some $y \in F(x)$. Without loss of generality we also have $z_{n} \rightarrow z$ for some $z \in C, z_{n} \in P_{C}\left(y_{n}\right)$ implies $z \in P_{C}(y)$, hence $P_{C}(y)=\{y\}$ yields $y_{n}-z_{n} \rightarrow 0$. Together with (4) this means $\widehat{z}_{n} \in F\left(x_{n}\right)+\widetilde{e}_{n}$ for some $\widetilde{e}_{n} \rightarrow 0$, hence 


$$
\begin{aligned}
x-h_{n} \widehat{z}_{n}=J_{h_{n}}\left(x-h_{n} \widehat{z}_{n}\right) & +h_{n} A_{h_{n}}\left(x-h_{n} \widehat{z}_{n}\right) \text { implies } \\
& x \in x_{n}+h_{n}\left(A x_{n}+F\left(x_{n}\right)+\widetilde{e}_{n}\right),
\end{aligned}
$$

i.e. (3) holds.

Rem arks 1. Specialized to the case of single-valued perturbations, the conditions on $F$ become " $F: \overline{D(A)} \rightarrow X$ continuous such that $A+F$ is accretive". In this situation the result is known and, using Lemma 1, it was first proved in [10] where it is Theorem 5.3. Independently, the same result was obtained in [13] Theorem II, by means of locally Lipschitz approximations of $F$. The first result about continuous perturbations of $m$ accretive operators is Theorem 1 in [1], where the assumptions on $F$ are $F: X \rightarrow X$ continuous and accretive. In the proof given there, it is shown that such an $F$ is in fact $s$-accretive, which means

$$
(F(x)-F(y), x-y)_{-} \geq 0 \quad \text { for all } x, y \in X
$$

hence $A+F$ is accretive. Let us note that $s$-accretivity of $F$ follows from the fact that $u^{\prime}=-F(u), u(0)=x$ has a unique $C^{1}$-solution on $\mathbb{R}_{+}$, for every $x \in X$. Hence $-F$ generates a semigroup of nonexpansive operators $S(t)$, given by $S(t) x:=u(t ; x)$, and therefore

$$
\begin{aligned}
(F(x)-F(y), x-y)_{-} & =\lim _{h \rightarrow 0+}\left(-\frac{S(h) x-x}{h}+\frac{S(h) y-y}{h}, x-y\right)_{-} \\
& \geq \underset{h \rightarrow 0+}{\lim _{h \rightarrow 0} h^{-1}|x-y|(|x-y|-|S(h) x-S(h) y|) \geq 0 .}
\end{aligned}
$$

In case $F: \overline{D(A)} \rightarrow X$ is continuous, accretive and satisfies the subtangential condition

$$
\lim _{h \rightarrow 0+} h^{-1} \rho(x+h F(x), \overline{D(A)})=0 \quad \text { on } \overline{D(A)},
$$

the same argument can be used to show that $F$ is $s$-accretive, since $u^{\prime}=-F(u), u(0)=x$ has a unique $C^{1}$-solution for every $x \in \overline{D(A)}$; see Remark 3 below. Hence $A+F$ is $m$ accretive, given that $A$ has this property. This is Theorem 2.8.1' in [12]. Without this additional boundary condition the result is not true; a counterexample is given in [13].

In the case of multivalued $F$ the situation is worse, since accretivity of $F$ is not sufficient then even if $F$ is defined on all of $X$. This is shown by the following

ExAmple 1. Let $X=\mathbb{R}^{2}$ with $|x|_{0}=\max \left\{\left|x_{1}\right|,\left|x_{2}\right|\right\}$ and $A: D(A) \rightarrow 2^{X} \backslash \emptyset$ be given by $A x=\mathbb{R} \times\{0\}$ on $D(A)=\{(s, s) \mid s \in \mathbb{R}\}$. Obviously, $R(I+\lambda A)=X$ for all $\lambda>0$. Moreover $A$ is accretive, since $x, y \in D_{A}, u \in A x, v \in A y$ means $x-y=(s, s)$ and $u-v=(h, 0)$ for some $s, h \in \mathbb{R}$, hence

$$
(u-v, x-y)_{+}=|s| \lim _{t \rightarrow 0+} t^{-1}(\max \{|s+t h|,|s|\}-|s|) \geq 0 .
$$

Let $F: X \rightarrow 2^{X} \backslash \emptyset$ be defined by

$$
F(x)= \begin{cases}\{(1,-1)\} & \text { if } x_{1}>x_{2} \\ \{(s,-s) \mid s \in[-1,1]\} & \text { if } x_{1}=x_{2} \\ \{(-1,1)\} & \text { if } x_{1}<x_{2}\end{cases}
$$


Evidently, $F$ is u.s.c. with compact convex values. Accretivity of $F$ can also be checked in a straight forward way, but we omit the details, since this $F$ is a special case of an example considered in [6]; see p. 296 there.

Now $A+F \equiv \mathbb{R} \times[-1,1]$ on $D(A)$ which is not accretive, since e.g. $x=(1,1)$, $y=(0,0), u=-x, v=y$ yield $(u-v, x-y)_{+}=-|x|_{0}^{2}=-1$.

2. For concrete applications, it would be useful to weaken the assumptions on $F$, since the values will often be only weakly compact and convex. We do not know how to prove a corresponding version of Theorem 1 in this case. If $F$ itself is $m$-accretive one can of course try to apply results about the sum of $m$-accretive operators like Theorem 3 in [14], saying that $A+F$ is $m$-accretive given that $A$ and $F$ have this property, $X$ and $X^{*}$ are uniformly convex and $D(A) \cap \operatorname{int}(D(F)) \neq \emptyset$.

4. Differential inclusions with dissipative right-hand side. By means of Theorem 1 we are going to obtain strong solutions of (1) if, among other assumptions, the $F(t, \cdot)$ are u.s.c. and satisfy a condition of dissipative type. Here $u$ is called strong solution of (1), if $u$ is absolutely continuous with $u(0)=x_{0}$ and a.e. differentiable such that $u^{\prime}(t) \in F(t, u(t))$ a.e. on $J$.

Let us first consider the special case when $F$ is given by $F(t, x)=-G(x)+w(t)$ on $J \times X$, where $w \in L_{X}^{1}(J)$ and $G: X \rightarrow 2^{X} \backslash \emptyset$ is accretive and u.s.c. with compact convex values. By Theorem 1 with $A=0$ we know that $G$ is $m$-accretive, hence (1) has a unique mild solution by Theorem 4.6 in [4]; see Remark 4 below for the definition of "mild solution". But in this situation $u$ is in fact a strong solution. This follows from the Proposition in [3], saying that every mild solution of $u^{\prime} \in-G(u)+w(t)$ is also a strong solution if $w \in L_{X}^{1}(J)$ and $G$ is weakly u.s.c. with closed domain and convex, weakly compact values; here weakly u.s.c. means $G^{-1}(A)$ closed for all weakly closed $A \subset X$.

The same conclusion holds if $G$ is only $\omega$-accretive, i.e.

$$
(y-\bar{y}, x-\bar{x})_{+} \geq-\omega|x-\bar{x}|^{2} \quad \text { for all } x, \bar{x} \in X, y \in G(x), \bar{y} \in G(\bar{x})
$$

with some $\omega \in \mathbb{R}$. Notice that the result mentioned above can be applied with $G+\omega I$ instead of $G$ and therefore the usual fixed point approach yields a strong solution for (1) with $F(t, x)=-G(x)+w(t)$. Let us record this information for later use.

Lemma 4. Let $X$ be a real Banach space, $G: X \rightarrow 2^{X} \backslash \emptyset$ be $\omega$-accretive for some $\omega \in \mathbb{R}$ and u.s.c. with compact convex values, $J=[0, a] \subset \mathbb{R}$ and $w \in L_{X}^{1}(J)$. Then the Cauchy problem

$$
u^{\prime} \in-G(u)+w(t) \quad \text { on } J, u(0)=x_{0}
$$

has a unique strong solution, for every $x_{0} \in X$.

We shall use Lemma 4 to prove a more general result which allows the right-hand side $F$ to depend on $(t, x)$ in a more complicated way. But still we need a rather strong assumption concerning the $t$-dependence. In the subsequent theorem we suppose that for every $\eta>0$ there exists a closed $J_{\eta} \subset J$ with $\mu\left(J \backslash J_{\eta}\right) \leq \eta$ such that the family $\left\{\left.F(\cdot, x)\right|_{J_{\eta}} \mid x \in X\right\}$ is locally equicontinuous, i.e. for every $x_{0} \in X$ there is $\delta=\delta\left(\eta, x_{0}\right)>$ 
0 such that the $F(\cdot, x)_{J_{\eta}}$ are equicontinuous for all $x \in B_{\delta}\left(x_{0}\right)$. If this holds we say that $\{F(\cdot, x) \mid x \in X\}$ is almost locally equicontinuous. Now we can prove

Theorem 2. Let $X$ be a real Banach space, $J=[0, a] \subset \mathbb{R}$ and let $F: J \times X \rightarrow 2^{X} \backslash \emptyset$ have compact convex values such that the following conditions hold.

(a) $\|F(t, x)\|:=\sup \{|y| \mid y \in F(t, x)\} \leq c(t)(1+|x|)$ on $J \times X$ with $c \in L^{1}(J)$.

(b) $(y-\bar{y}, x-\bar{x})_{-} \leq k(t)|x-\bar{x}|^{2}$ for all $t \in J, x, \bar{x} \in X, y \in F(t, x), \bar{y} \in F(t, \bar{x})$ with $k \in L^{1}(J)$.

(c) $F(t, \cdot)$ is u.s.c. for almost all $t \in J$.

(d) The family of maps $\{F(\cdot, x): x \in X\}$ is almost locally equicontinuous.

Then initial value problem (1) has a unique strong solution on $J$.

Proof. (1). Let us first reduce to the case $c(t) \equiv k(t) \equiv 1$. For this purpose define $\varphi \in L^{1}(J)$ by $\varphi=\max \{1, c, k\}$. The $\operatorname{map} t \rightarrow \int_{0}^{t} \varphi(s) d s$ from $J$ to $\widetilde{J}:=\left[0,|\varphi|_{1}\right]$ is continuous and strictly increasing. Let $\phi$ be its inverse and define $\widetilde{F}: \widetilde{J} \times X \rightarrow 2^{X} \backslash \emptyset$ by

$$
\widetilde{F}(t, x)=\frac{1}{\varphi(\phi(t))} F(\phi(t), x) \quad \text { for }(t, x) \in \widetilde{J} \times X .
$$

Evidently, $u$ is a solution of (1) iff $v(t):=u(\phi(t))$ is a solution of (1) with $F$ and $J$ replaced by $\widetilde{F}$ and $\widetilde{J}$, respectively. It is easy to check that $\widetilde{F}$ has properties (a)-(c) with $c(t) \equiv k(t) \equiv 1$ on $\widetilde{J} \times X$. To see that $\widetilde{F}$ also satisfies $(\mathrm{d})$, let $\eta>0$ be given. Then there is $\sigma=\sigma(\eta)>0$ such that $\mu(A) \leq \sigma$ implies $\int_{A} \varphi(t) d t \leq \eta$ for every Lebesgue measurable $A \subset J$. Exploitation of condition (d) for $F$ yields a closed $J_{\sigma} \subset J$ with $\mu\left(J \backslash J_{\sigma}\right) \leq \sigma$ such that the family $\left\{\left.F(\cdot, x)\right|_{J_{\sigma}} \mid x \in X\right\}$ is locally equicontinuous and $F(t, \cdot)$ is u.s.c. for all $t \in J_{\sigma}$. Since $\varphi$ has the Lusin property, we may also assume that $\varphi_{J_{\sigma}}$ is continuous. Let $\widetilde{J}_{\eta}:=\phi^{-1}\left(J_{\sigma}\right)$. Using the fact that $J_{\sigma}$ is closed it is easy to check that $\mu\left(\widetilde{J}_{\eta}\right)=\int_{J_{\eta}} \varphi(t) d t$, hence $\mu\left(\widetilde{J} \backslash \widetilde{J}_{\eta}\right) \leq \eta$. Now we are done, since

$$
d_{H}(\widetilde{F}(t, x), \widetilde{F}(s, x)) \leq\left|\frac{1}{\varphi(\phi(t))}-\frac{1}{\varphi(\phi(s))}\right|(1+|x|)+d_{H}(F(\phi(t), x), F(\phi(s), x)),
$$

$\left\{\left.F(\phi(\cdot), x)\right|_{\widetilde{J}_{\eta}} \mid x \in X\right\}$ is locally equicontinuous and $\left.\left(\frac{1}{\varphi(\phi(\cdot))}\right)\right|_{\widetilde{J}_{\eta}}$ is uniformly continuous. In the sequel we will denote $\widetilde{F}$ and $\widetilde{J}$ by $F$ and $J$ again.

(2). Given $\eta>0$, let $J_{\eta} \subset J$ be closed with $\mu\left(J \backslash J_{\eta}\right) \leq \eta$ such that the family of maps $\left\{\left.F(\cdot, x)\right|_{J_{\eta}} \mid x \in X\right\}$ is locally equicontinuous, where we may assume $\{0, a\} \subset J_{\eta}$. Then $J \backslash J_{\eta}=\bigcup_{n \geq 1}\left(\alpha_{n}, \beta_{n}\right)$ for disjoint $\left(\alpha_{n}, \beta_{n}\right) \subset J$, since $J \backslash J_{\eta}$ is open. Let $F_{\eta}: J \times X \rightarrow$ $2^{X} \backslash \emptyset$ be defined by

$$
F_{\eta}(t, x)= \begin{cases}F(t, x) & \text { if } t \in J_{\eta} \\ F\left(\alpha_{n}, x\right) & \text { if } t \in\left(\alpha_{n}, \beta_{n}\right) \text { for some } n \geq 1\end{cases}
$$

Then $F_{\eta}$ has compact convex values, satisfies (a), (b) with $c(t) \equiv k(t) \equiv 1$ and $F_{\eta}(t, \cdot)$ is u.s.c. for all $t \in J$. We want to show that (1) with $F_{\eta}$ instead of $F$ has a strong solution. For this purpose let us first prove that $(1)$ with $F$ replaced by $F_{\eta}$ has an $\varepsilon$-approximate 
solution $u=u_{\varepsilon}$ for every $\varepsilon \in(0,1)$, by which we mean

$$
\begin{array}{r}
u(t)=x_{0}+\int_{0}^{t} w(s) d s \text { on } J \text { with } w \in L_{X}^{1}(J) \\
\text { such that } \int_{0}^{a} \rho\left(w(t), F_{\eta}(t, u(t))\right) d t \leq \varepsilon a .
\end{array}
$$

This will be done by using Zorn's Lemma. But notice first that there is $R>1$ such that every $u$ satisfying (5) for some $\varepsilon \in(0,1)$ has $|u|_{0} \leq R-1$. Therefore, we can obtain approximate solutions such that also $\left|u^{\prime}(t)\right| \leq R$ a.e. on $J$. Consider the set

$$
\begin{aligned}
M=\{(u, h) \mid h & \in(0, a], u:[0, h] \rightarrow X \text { satisfies }(5) \text { with } \\
& J \text { replaced by }[0, h] \text { such that }|w(t)| \leq R \text { a.e. on }[0, h]\},
\end{aligned}
$$

equipped with the partial ordering $(u, h) \leq(\bar{u}, \bar{h})$ if $h \leq \bar{h}$ and $u(t)=\bar{u}(t)$ on $[0, h]$. Let us show $M \neq \emptyset$. There is $\delta=\delta\left(\eta, x_{0}\right)>0$ such that $\left\{\left.F(\cdot, x)\right|_{J_{\eta}} \mid x \in B_{\delta}\left(x_{0}\right)\right\}$ is equicontinuous. Hence there is $h_{0}>0$ such that $d_{H}(F(0, x), F(t, x)) \leq \varepsilon$ for every $t \in\left[0, h_{0}\right] \cap J_{\eta}$ and every $x \in B_{\delta}\left(x_{0}\right)$. By the definition of $F_{\eta}$ this implies

$$
d_{H}\left(F_{\eta}(0, x), F_{\eta}(t, x)\right) \leq \varepsilon \quad \text { for every } t \in\left[0, h_{0}\right], x \in B_{\delta}\left(x_{0}\right) .
$$

Let $u$ be the strong solution of the initial value problem

$$
u^{\prime} \in F_{\eta}(0, u) \quad \text { on } J, u(0)=x_{0},
$$

which exists due to Lemma 4 with $G:=-F_{\eta}(0, \cdot)$ and $w:=0$. Since there is $h \in\left(0, h_{0}\right]$ such that $\left|u(t)-x_{0}\right| \leq \delta$ on $[0, h]$, estimate (6) implies

$$
\int_{0}^{h} \rho\left(u^{\prime}(t), F_{\eta}(t, u(t))\right) d t \leq \int_{0}^{h} d_{H}\left(F_{\eta}(0, u(t)), F_{\eta}(t, u(t))\right) d t \leq \varepsilon h .
$$

Hence $|u(t)| \leq R-1$ on $[0, h]$, which implies $\left|u^{\prime}(t)\right| \leq\left\|F_{\eta}(0, u(t))\right\| \leq R$ a.e. on $[0, h]$, and therefore $(u, h) \in M$. It is obvious that every ordered subset of $M$ has an upper bound, hence $M$ has a maximal element $\left(u^{*}, h^{*}\right)$ by Zorn's Lemma. Moreover $h^{*}=a$ since otherwise we may repeat the argument given above with $\left(h^{*}, u^{*}\left(h^{*}\right)\right)$ instead of $\left(0, x_{0}\right)$ to get an $\varepsilon$-approximate solution on $\left[0, h^{*}+h\right]$ which extends $u^{*}$, a contradiction.

(3). Now let $\left(\varepsilon_{k}\right) \subset(0,1)$ satisfy $\varepsilon_{k} \rightarrow 0+$ and $u_{k}$ be $\varepsilon_{k}$-approximate solutions of (1) for $F_{\eta}$. Then, for fixed $m$ and $n, \psi(t)=\left|u_{n}(t)-u_{m}(t)\right|$ satisfies $\psi(0)=0$ and

$\psi(t) \psi^{\prime}(t)=\left(u_{n}^{\prime}(t)-u_{m}^{\prime}(t), u_{n}(t)-u_{m}(t)\right)_{-} \leq\left(\rho_{n}(t)+\rho_{m}(t)\right) \psi(t)+\psi(t)^{2} \quad$ a.e. on $J$, where $\rho_{k}(t)=\rho\left(u_{k}^{\prime}(t), F_{\eta}\left(t, u_{k}(t)\right)\right)$ on $J$. This implies $e^{-a}|\psi|_{0} \leq\left|\rho_{n}\right|_{1}+\left|\rho_{m}\right|_{1} \leq a\left(\varepsilon_{n}+\right.$ $\left.\varepsilon_{m}\right)$. Consequently, $\left(u_{k}\right)$ is a Cauchy sequence in $C_{X}(J)$, hence $\left|u_{k}-u\right|_{0} \rightarrow 0$ for some $u \in C_{X}(J)$ with $u(0)=x_{0}$; notice that $\left(u_{k}\right)$ is equicontinuous. Since $F_{\eta}(t, \cdot)$ is u.s.c. with compact values for all $t \in J$, the sets $F_{\eta}\left(t,\left\{\overline{u_{k}(t) \mid k \geq 1}\right\}\right)$ are compact. By Lemma 3 we may therefore assume $w_{k}=u_{k}^{\prime} \rightarrow w$ for some $w \in L_{X}^{1}(J)$. Together with $u_{k} \rightarrow u$ in $C_{X}(J)$ this implies $u(t)=x_{0}+\int_{0}^{t} w(s) d s$ on $J$. By Mazur's Theorem there are $\bar{w}_{k} \in \operatorname{conv}\left\{w_{j} \mid j \geq k\right\}$ with $\bar{w}_{k} \rightarrow w$ in $L_{X}^{1}(J)$, hence w.l.o.g. $\bar{w}_{k}(t) \rightarrow w(t)$ a.e. on $J$ by passing to a certain subsequence. Let $J_{0}=\left\{t \in J \mid w_{k}(t) \in F_{\eta}\left(t, u_{k}(t)\right)\right.$ for all $k \geq 1$, $\left.\bar{w}_{k}(t) \rightarrow w(t)\right\}$ and $t \in J_{0}$. Then, given $\sigma>0$, we have $w_{k}(t) \in F_{\eta}(t, u(t))+B_{\sigma}(0)$ for all 
large $k$, hence the same for $\bar{w}_{k}(t)$. Evidently, this implies $w(t) \in F_{\eta}(t, u(t))$ on $J_{0}$, hence a.e. on $J$ and therefore $u$ is a strong solution of (1) with $F_{\eta}$.

4. Let $\eta_{k} \searrow 0$ and $J_{k}:=J_{\eta_{k}}, F_{k}:=F_{\eta_{k}}$ be given as in step 2, where we may assume $J_{k} \subset J_{k+1}$ for $k \geq 1$. By the previous step, initial value problem (1) with $F_{k}$ instead of $F$ has a solution $u_{k}$ for every $k \geq 1$. Moreover, $\left|u_{k}\right|_{0} \leq R$ for all $k \geq 1$ with some $R>0$, since all $F_{k}$ satisfy (a) with $c(t) \equiv 1$. For fixed $m \geq 1$, we have $F_{n}=F$ on $J_{m} \times X$ for all $n \geq m$, hence $\psi(t):=\left|u_{n}(t)-u_{m}(t)\right|$ has

$$
\psi^{\prime}(t) \leq \psi(t) \chi_{J_{m}}(t)+2(1+R) \chi_{J \backslash J_{m}}(t) \quad \text { a.e. on } J, \psi(0)=0,
$$

for those $n$. Therefore, application of Gronwall's Lemma shows that $\left(u_{k}\right)$ is Cauchy in $C_{X}(J)$. Hence $\left|u_{k}-u\right|_{0} \rightarrow 0$ for some $u \in C_{X}(J)$ with $u(0)=x_{0}$, and $u^{\prime}(t) \in F(t, u(t))$ a.e. on $J$ can be seen as in step 3. So, we have shown that (1) has a strong solution. Evidently we are done, since uniqueness is an obvious consequence of (b).

Additional information is contained in the following

Rem arks 3. If $X$ is a real Hilbert space condition $(\mathrm{d})$ can be replaced by " $F(\cdot, x)$ has a strongly measurable selection" and the values of $F$ need only be closed convex. This is Theorem 10.5 in [7], and Theorem 2 is a first step to extend this result to general Banach spaces. Therefore this gives a partial answer to Problem 10.6 in [7].

Let us also mention that, specialized to the single-valued case, conditions (a) and (d) hold in case $F$ is almost continuous, which is the same as " $F$ is measurable in $t$ and continuous in $x$ " for separable $X$. For continuous single-valued $F$ a corresponding version of Theorem 2 holds even if the maps $F(t, \cdot)$ are only defined on time-dependent sets $D(t) \subset X$, given that $\operatorname{gr}(D)$ is closed from the left and $F$ also satisfies the subtangential condition

$$
\lim _{h \rightarrow 0+} h^{-1} \rho(x+h F(t, x), D(t+h))=0 \quad \text { for all } t \in[0, a), x \in D(t) ;
$$

see Theorem 3 in [9]. For multivalued and almost u.s.c. right-hand sides, such an existence result under time-dependent constraints holds if the condition (b) of dissipative type is replaced by a certain compactness assumption. The details concerning the latter case can be found in $[5]$.

4. A different approach to prove a result like Theorem 2 is to get first the existence of a mild solution and then to show that it is in fact a strong solution; remember the proof of Lemma 4. By a mild solution $u$ of (1) one means $u \in C_{X}(J)$ being the uniform limit of a sequence of approximate solutions $u_{m}$ (corresponding to a sequence $\varepsilon_{m} \rightarrow 0+$ ) which solve an implicit difference scheme. More precisely, $v$ is such an approximate solution corresponding to $\varepsilon>0$ if there are $x_{1}, \ldots, x_{n+1} \in X$ and a partition $0=t_{0}<t_{1}<\ldots<$ $t_{n} \leq t_{n+1}=a$ of $J$ such that, for all $k=0, \ldots, n$, one has:

$$
\begin{array}{ll}
t_{k+1}-t_{k} \leq \varepsilon, & v(t)=x_{k} \text { on }\left[t_{k}, t_{k+1}\right) \text { and } \\
\frac{x_{k+1}-x_{k}}{t_{k+1}-t_{k}} \in F\left(t_{k+1}, x_{k+1}\right)+z_{k} & \text { with }\left|z_{k}\right| \leq \varepsilon .
\end{array}
$$

Now, under the conditions of Theorem 2 where w.l.o.g. $k(t) \equiv \omega$, it is easy to see that we get such approximate solutions, since almost all $-F(t, \cdot)$ are $m$ - $\omega$-accretive. In fact one 
only needs condition (3) with $B$ replaced by $F(t+h, \cdot)$; see e.g. Chapter 1.3 .5 in [12]. Then the main problem is to obtain the uniform convergence of $\left(v_{m}\right)$, and one may try to apply results about time-dependent $\omega$-accretive operators like Theorem 3.5 in [11]. Specialized to the situation under consideration, this theorem guarantees that $\left|v_{m}-u\right|_{0} \rightarrow$ 0 for some $u \in C_{X}(J)$, given that

$$
(y, x-\bar{x})_{-}+(-\bar{y}, x-\bar{x})_{-} \leq \omega|x-\bar{x}|^{2}+\varphi(t, \bar{t})|x-\bar{x}|
$$

for all $t, \bar{t} \in J, x, \bar{x} \in X, y \in F(t, x)$ and $\bar{y} \in F(\bar{t}, \bar{x})$ with some $\omega \geq 0$ and a bounded upper semicontinuous symmetric function $\varphi: J \times J \rightarrow \mathbb{R}_{+}$satisfying

$$
\lim _{r \rightarrow 0+} \sup \{\varphi(t, \bar{t})|| t-\bar{t} \mid \leq r\}=0 \quad \text { on } J \times J .
$$

It is sufficient that this condition holds locally, i.e. for all $x, \bar{x} \in B_{\delta}(\widehat{x})$ for every $\widehat{x} \in X$ and some $\delta=\delta(\widehat{x})>0$, where $\omega$ and $\varphi$ may depend on $B_{\delta}(\widehat{x})$. In the situation described in Theorem 2 it is not clear if this condition is satisfied, but it holds if $k(t) \equiv \omega$ in (b) and the maps $F(\cdot, x)$ are locally equicontinuous. In this case, once the existence of mild solutions of (1) is established, the proof is easily finished: given $v_{m} \rightarrow u$ in $C_{X}(J)$, consider functions $u_{m}$ being linear on each $\left[t_{k}^{m}, t_{k+1}^{m}\right]$ with $u_{m}\left(t_{k}^{m}\right):=v_{m}\left(t_{k}^{m}\right)$. Evidently $\left|u_{m}-u\right|_{0} \rightarrow 0$. Then $u_{m}^{\prime} \rightarrow w$ in $L_{X}^{1}(J)$ and $u^{\prime}(t)=w(t) \in F(t, u(t))$ a.e. on $J$ can be proved similar to step 3 of the proof of Theorem 2 .

Acknowledgment. The first part of this work was completed while the author was visiting the Université de Franche-Comté (Besançon). It is a pleasure to thank the Equipe de Mathématiques de Besançon for its kind hospitality, in particular Professor Ph. Benilan for helpful discussions and suggestions.

\section{References}

[1] V. Barbu, Continuous perturbations of nonlinear $m$-accretive operators in Banach spaces, Boll. Un. Mat. Ital. (4) 6 (1972), 270-278.

[2] V. Barbu, Nonlinear Semigroups and Differential Equations in Banach Spaces, Noordhoff, Leyden 1976.

[3] L. Barthelemy, Equivalence entre bonne solution et solution forte d'une équation d'évolution gouvernée par un opérateur s.c.s, Publ. Math. Fac. Sci. Besançon, Anal. Non Linéaire, 1985-86, 9 (1986), 3-8.

[4] Ph. Benilan, M. G. Crandall and A. Pazy, Nonlinear Evolution Equations in Banach Spaces. (book in preparation).

[5] D. Bothe, Multivalued differential equations with time-dependent constraints, Proc. of the 1. World Congress of Nonlinear Analysts (to appear).

[6] M. G. Crandall and T. M. Liggett, Generation of semi-groups of nonlinear transformations on general Banach spaces, Amer. J. Math. 93 (1971), 265-298.

[7] K. Deimling, Multivalued Differential Equations, W. De Gruyter, Berlin 1992.

[8] J. Diestel, W. M. Ruess and W. Schachermayer, Weak compactness in $L^{1}(\mu, X)$, Proc. Amer. Math. Soc. 118 (1993), 447-453.

[9] N. Kenmochi and T. Takahashi Nonautonomous differential equations in Banach spaces, Nonlinear Analysis, 4 (1980), 1109-1121. 
[10] Y. Kobayashi, Difference approximation of Cauchy problems for quasi-dissipative operators and generation of nonlinear semigroups, J. Math. Soc. Japan 27 (1975), 640-665.

[11] K. Kobayasi, Y. Kobayashi and S. Ohar u, Nonlinear evolution operators in Banach spaces, Osaka J. Math. 21 (1984), 281-310.

[12] N. H. Pavel, Nonlinear Evolution Operators and Semigroups. Lect. Notes Math. 1260, Springer 1987.

[13] M. Pierre, Perturbations localement Lipschitziennes et continues d'opérateurs m-accretifs, Proc. Amer. Math. Soc. 58 (1976), 124-128.

[14] J. Prüss, A characterization of uniform convexity and applications to accretive operators, Hiroshima Math. J. 11 (1981), 229-234. 